\title{
Stochastic-dynamical thermostats for constraints and stiff restraints
}

\author{
J. Bajars ${ }^{1, a}$, J. Frank ${ }^{1, b}$, and B. Leimkuhler ${ }^{2, c}$ \\ 1 Centrum Wiskunde \& Informatica (CWI), PO Box 94079, 1090 GB Amsterdam, \\ The Netherlands \\ 2 School of Mathematics and Maxwell Institute for Mathematical Sciences, The \\ University of Edinburgh, James Clerk Maxwell Building, The King's Buildings, \\ Mayfield Road, Edinburgh, Scotland EH9 3JZ, UK
}

Received 09 September 2011 / Received in final form 10 October 2011 Published online 13 December 2011

\begin{abstract}
A broad array of canonical sampling methods are available for molecular simulation based on stochastic-dynamical perturbation of Newtonian dynamics, including Langevin dynamics, Stochastic Velocity Rescaling, and methods that combine Nosé-Hoover dynamics with stochastic perturbation. In this article we discuss several stochasticdynamical thermostats in the setting of simulating systems with holonomic constraints. The approaches described are easily implemented and facilitate the recovery of correct canonical averages with minimal disturbance of the underlying dynamics. For the purpose of illustrating our results, we examine the numerical application of these methods to a simple atomic chain, where a Fixman term is required to correct the thermodynamic ensemble.
\end{abstract}

\section{Introduction}

Constraints are used in diverse ways in molecular dynamics studies. They replace the stiffest bond stretches in biomolecular models, allowing simulation with larger timesteps than would otherwise be possible [5]; they are part of free-energy and reaction pathway techniques $[6-8]$, and they are used to constrain normal modes in some enhanced sampling approaches [3]. In general, these methods are implemented in the setting of canonical sampling, i.e. with thermostats, or barostats. The proper treatment of constraints in combination with appropriate thermostating devices is therefore of great importance and the neglect of their correct handling may lead to uncontrollable errors in computed observables.

When constraints are introduced as a modelling device, they effectively reduce the dimension of phase space. To achieve a good agreement in thermodynamical calculations, a free energy correction, representing the energy of the missing degrees of freedom, should be incorporated (e.g. through a Fixman potential $[4,10,15]$ ); this

\footnotetext{
a e-mail: janis.bajars@cwi.nl

b e-mail: jason@cwi.nl

c e-mail: b.leimkuhler@ed.ac.uk
} 
thermodynamic correction (which can only be realized in a canonical simulation, i.e. with a thermostat) has the potential to interfere with the calculation of dynamical properties such as diffusion rates. In this article we discuss the use of stochasticdynamical techniques for treating constrained models in the context of these issues.

Consider a Hamiltonian system with generalized coordinates $q, p \in \mathbf{R}^{n}$ and Hamiltonian

$$
H(q, p)=\frac{1}{2} p^{T} M^{-1} p+V(q),
$$

where $M$ is a positive definite and symmetric (typically diagonal) mass matrix. The equations of motion are

$$
\begin{gathered}
\frac{d q}{d t}=M^{-1} p, \\
\frac{d p}{d t}=-\nabla V(q) .
\end{gathered}
$$

The Hamiltonian represents the total energy and is a first integral of (2)-(3). Consequently, a trajectory of this system with initial condition $\left(q_{0}, p_{0}\right)$ samples the constant energy surface $H(q, p)=H_{0} \equiv H\left(q_{0}, p_{0}\right)$, and when the flow is sufficiently ergodic, we expect time averages to converge to ensemble averages in the microcanonical ensemble

$$
\rho_{\mu}(q, p)=Z_{\mu}^{-1} \delta\left(H(q, p)-H_{0}\right), \quad Z_{\mu}=\int \delta\left(H(q, p)-H_{0}\right) d q \wedge d p,
$$

where $d q \wedge d p=d q_{1} \wedge \cdots \wedge d q_{n} \wedge d p_{1} \wedge \cdots \wedge d p_{n}$ is the volume form on $\mathbf{R}^{2 n}$.

In molecular dynamics one is often interested, not in the dynamics of an isolated system at constant energy, but in a system in thermal equilibrium with a reservoir at temperature $\beta^{-1}=k_{B} T$. In this case an ergodic system should sample the canonical (Gibbs) distribution

$$
\rho_{\beta}(q, p)=Z^{-1} e^{-\beta H(q, p)}, \quad Z=\int e^{-\beta H(q, p)} d q \wedge d p .
$$

A wide variety of thermostatting devices have been proposed to perturb the Hamiltonian dynamics (2)-(3) in order to sample the canonical distribution (5). In Section 2 of this article, we will discuss various schemes and the relationships among them, and mention a recently proposed unified framework.

In Section 3 of this paper we will generalize the discussion to the case in which the dynamics (2)-(3) is subjected to a holonomic constraint, i.e. we enforce an algebraic relation on the position variables $q$, i.e.

$$
g(q)=0, \quad g: \mathbf{R}^{n} \rightarrow \mathbf{R}^{m} .
$$

This constraint restricts the positions $q$ to an $n-m$ dimensional manifold $\mathcal{M}$, and implies a restriction of the velocities $M^{-1} p$ to the tangent space $T_{q} \mathcal{M}$, i.e.

$$
\nabla g(q) M^{-1} p=0
$$

which follows by taking the derivative of (6) with respect to $t$ along a trajectory.

The constraint is enforced by introducing a Lagrange multiplier $\lambda \in \mathbf{R}^{m}$

$$
\begin{aligned}
\frac{d q}{d t} & =M^{-1} p, \\
\frac{d p}{d t} & =-\nabla V(q)-\nabla g(q)^{T} \lambda, \\
0 & =g(q)
\end{aligned}
$$


with the augmented Hamiltonian

$$
\tilde{H}(q, p, \lambda)=\frac{1}{2} p^{T} M^{-1} p+V(q)+g(q)^{T} \lambda .
$$

Taking the second derivative of (6) with respect to time and making use of (9) yields an explicit expression for the Lagrange multiplier

$$
\lambda=\left(\nabla g(q) M^{-1} \nabla g(q)^{T}\right)^{-1}\left(\nabla g(q) M^{-1} \nabla V(q)-G(q)\left(M^{-1} p, M^{-1} p\right)\right),
$$

where $G(q)$ is the symmetric three-tensor (Hessian) of partial derivatives of $\nabla g(q)$, whose contraction is denoted $G(q)(\cdot, \cdot)$.

When the flow of the constrained dynamical system (8)-(10) is sufficiently ergodic, we expect time averages to converge to ensemble averages in the microcanonical ensemble

$$
\begin{aligned}
& \rho_{\mu, c}=Z_{\mu, c}^{-1} \delta\left(H(q, p)-H_{0}\right) \delta(g(q)) \delta\left(\nabla g(q) M^{-1} p\right), \\
& Z_{\mu, c}^{-1}=\int \delta\left(H(q, p)-H_{0}\right) \delta(g(q)) \delta\left(\nabla g(q) M^{-1} p\right) d q \wedge d p .
\end{aligned}
$$

In the context of molecular dynamics when one is interested in a system in thermal equilibrium with a reservoir at temperature $\beta^{-1}$, an ergodic system should sample the hybrid (Gibbs) distribution

$$
\begin{aligned}
& \rho_{\beta, c}(q, p)=Z_{c}^{-1} e^{-\beta H(q, p)} \delta(g(q)) \delta\left(\nabla g(q) M^{-1} p\right), \\
& Z_{c}=\int e^{-\beta H(q, p)} \delta(g(q)) \delta\left(\nabla g(q) M^{-1} p\right) d q \wedge d p .
\end{aligned}
$$

Some numerical methods for implementing constrained sampling methods discussed in Sect. 3 are provided in the appendix.

Thermostats are, by their very nature, artificial devices. The purposes of thermostatting are varied, including the efficient decorrelation of sampling trajectories and the correction of temperature perturbations due to numerical drift [23,24] or even applied forcing [14]. A strong motivation for some of the recent proposals (in particular $[1,2,22])$ for thermostats has been the desire to control temperature while exerting the least influence on the dynamics of the system, i.e. staying as close as possible to microcanonical dynamics. This topic has been studied in detail in a recent article [19]. If the convergence to equilibrium of two methods is similar, then the problem is to compare the accuracy of autocorrelation functions produced by the methods, as a measure of the efficiency of the thermostat. That is, for a given rate of convergence to the equilibrium measure, a more efficient thermostat is one that least perturbs the dynamics (measured in terms of auto-correlation functions). Similarly, we say that a thermostat is gentle if its effect on dynamics is relatively mild for a given rate of convergence of the measure. In this vein, we here demonstrate in Sect. 4 that results of [19] on the smaller autocorrelation error of the Nosé-Hoover-Langevin method compared to Langevin dynamics carry over to the constrained setting.

In Sect. 5 we consider the situation in which constraints are introduced as modelling devices derived as limits of strong restraints. By restraints we mean stiffly oscillatory forces or soft constraints. We have in mind applications in molecular dynamics where the constraints are used as models for chemical bonds which should, in a somewhat more accurate model, be allowed to stretch. The suppression of these fast bond vibrations has an advantage for numerical integration: the fast vibration necessitates a small timestep which is not in fact needed to resolve the expensive components of the molecular force field (such as Coulombic interactions). However, this simple approach has a fundamental problem: the thermodynamic properties of a system with strong restraint are not equivalent to those of the constrained system. 
We can see this by considering the fact that the model with restraint, however stiff, still has momenta which sample a Boltzmann distribution, i.e. they are normally distributed, whereas the constrained system cannot have this property due to the associated tangent space constraint. The energy which would be equidistributed into the transverse components to the constraint manifold must be accounted for in the model using a Fixman biasing potential $[4,10,15,16,25,26]$.

The methods are applied to a small chain of 4 atoms with Lennard-Jones forces. Our results suggest that Langevin dynamics performs in a reliable and robust manner for the computation of (stationary) thermodynamic averages, but it is unable to recover autocorrelation functions accurately. In our example, the Nosé-HooverLangevin method and Stochastic Velocity Rescaling method prove superior to the Langevin method when the goal is the calculation of dynamics.

\section{Stochastic-dynamical thermostats}

In this section, we discuss and compare a variety of methods for achieving canonical sampling in the unconstrained setting. All of these can be written in a simple unified framework [18].

Thermostats come in many different varieties, designed for a range of different purposes. Sometimes thermal control is effected by means of a randomized step with a Metropolis (Monte-Carlo) accept/reject step. In this article we are only concerned with methods that generate sampling paths by discretization of a suitable stochastic differential equation obtained as a perturbation of the original dynamics. One of the most popular methods is Langevin dynamics defined by

$$
\begin{aligned}
& d q=M^{-1} p d t \\
& d p=-\nabla V(q) d t-\frac{\beta}{2} \sigma \sigma^{T} M^{-1} p d t+\sigma d W,
\end{aligned}
$$

where $W(t)$ is a vector of independent Wiener processes in $\mathbf{R}^{n}$ and $\sigma \in \mathbf{R}^{n \times n}$.

Nosé-Hoover-Langevin (NHL) dynamics $[20,22]$ is defined by

$$
\begin{aligned}
& d q=M^{-1} p d t, \\
& d p=-\nabla V(q) d t+\xi p d t, \\
& d \xi=\frac{1}{\alpha}\left(n-\beta p^{T} M^{-1} p\right) d t-\gamma \xi d t+\sigma d w,
\end{aligned}
$$

where $\xi \in \mathbf{R}$ is an auxiliary thermostat variable and $w(t)$ is a scalar Wiener process, $\sigma \in \mathbf{R}$ and $\gamma=\alpha \sigma^{2} / 2$. The NHL method is constructed such that the extended measure

$$
\hat{\rho}(q, p, \xi)=\rho_{\beta}(q, p) \rho_{\alpha}(\xi)
$$

is stationary under the phase space flow, where $\rho_{\alpha}(\xi)$ is the mean-zero normal distribution with variance $\alpha^{-1}$ :

$$
\rho_{\alpha}(\xi)=\sqrt{\frac{\alpha}{2 \pi}} \exp \left(-\alpha \frac{\xi^{2}}{2}\right) .
$$

The NHL dynamics can furthermore be shown to be ergodic in the measure (20) whenever the Lie algebra generated by $p$ and $\nabla V(q)$ spans $\mathbf{R}^{n}$. Whence the projected dynamics on $\mathbf{R}^{2 n}$ ergodically samples (5). 
In [19] it was shown that the NHL dynamics allows a more accurate computation of velocity auto-correlation functions (VAF) in the asymptotic limit of small correlation times.

Other schemes have been suggested recently for sampling purposes. Like NHL, the Stochastic Velocity Rescaling (SVR) method of Bussi et al. [1,2] has been suggested to provide for thermostatting with a weak perturbation of dynamics. This claim was verified analytically by [19] who generalized the method to:

$$
\begin{aligned}
& d q=\nabla_{p} H d t, \\
& d p=-\nabla_{q} H d t-\Psi(K) p d t+\sqrt{2 k_{B} T \Phi(K)} p d W,
\end{aligned}
$$

where $W(t)$ is a (scalar) Wiener process, and $\Phi, \Psi$ are related by

$$
\Psi(K)=\left(2 K-(1+n) k_{B} T\right) \Phi(K)-2 k_{B} T K \frac{d \Phi}{d K} .
$$

With these choices, the method can be shown to preserve the Gibbs distribution $\rho_{\beta}$. For the SVR method to be well defined, one also assumes

$$
\begin{gathered}
K \Phi(K) \text { is bounded as } K \rightarrow 0, \\
\Phi(K) \text { grows at most polynomially as } K \rightarrow \infty .
\end{gathered}
$$

Frank \& Gottwald [11] have shown that the NHL method converges to SVR (22)-(23) in an appropriate strong perturbation limit $\alpha \rightarrow 0$.

We mention that all the various methods described in this section have been unified into a single general formulation [18] which can be viewed as including any canonical measure-preserving deterministic extensions of the equations of motion coupled with measure-preserving stochastic perturbation. In general, one augments the system by some degrees of freedom $\xi_{1}, \xi_{2}, \ldots, \xi_{k}$ and designs an extended dynamics so that the density $\rho_{\beta} \hat{\rho}\left(\xi_{1}, \xi_{2}, \ldots, \xi_{k}\right)$ is preserved, for some suitable choice of $\hat{\rho}$. Then, if ergodic, the extended system can be used to compute canonical phase space averages with respect to $\rho_{\beta}$ (essentially by averaging out over the auxiliary degrees of freedom).

\section{Extension of stochastic thermostats to holonomic constraints}

In this section we discuss various methods for treating the equations of motion with holonomic constraints, including Langevin dynamics, the Nosé-Hoover-Langevin dynamics and the Stochastic Velocity Rescaling thermostats. Besides the added constraints and associated Lagrange multiplier, the main difference in the methods is the reduction of degrees of freedom from $n$ to $n-m$, which appears explicitly in the thermostat relations. The derivations are included in Appendix A.

The positions of the system (8)-(10) are constrained to the configuration manifold $\mathcal{M}$ of co-dimension $m$ :

$$
\mathcal{M}=\left\{q \in \mathbf{R}^{n} \mid g(q)=0\right\},
$$

and the associated phase space is the tangent bundle denoted by

$$
\mathcal{T} \mathcal{M}=\left\{q, p \in \mathbf{R}^{n} \mid q \in \mathcal{M}, \nabla g(q) M^{-1} p=0\right\} .
$$

For a given $q \in \mathcal{M}$, the tangent space is defined by

$$
\mathcal{T}_{q} \mathcal{M}=\left\{p \in \mathbf{R}^{n} \mid \nabla g(q) M^{-1} p=0\right\} .
$$


Given a measure (5) on the base space $\mathbf{R}^{2 n}$, the associated measure on the tangent bundle $T \mathcal{M}$ is obtained by restricting the volume form $d q \wedge d p$ to $T \mathcal{M}$. Following [17] we introduce a local chart $(\zeta, \eta)$, where $\zeta, \eta \in \mathcal{D} \subset \mathbf{R}^{n-m}$ and a mapping $\phi(\zeta)$ : $\mathcal{D} \rightarrow \mathbf{R}^{n}$ satisfying $g(\phi(\zeta))=0$ and $\nabla g(q) \nabla \phi=0$. We parametrize $T_{q} \mathcal{M}$ using the relations

$$
\begin{aligned}
& q=\phi(\zeta), \\
& p=\nabla \phi\left(\nabla \phi^{T} \nabla \phi\right)^{-1} \eta .
\end{aligned}
$$

Here we assume that the square matrix $\nabla \phi^{T} \nabla \phi$ has full rank. This map is a canonical transformation. The Hamiltonian (11) is transformed to

$$
\hat{H}(\zeta, \eta)=\frac{1}{2} \eta^{T}\left(\nabla \phi^{T} \nabla \phi\right)^{-T}\left(\nabla \phi^{T} M^{-1} \nabla \phi\right)\left(\nabla \phi^{T} \nabla \phi\right)^{-1} \eta+V(\phi(\zeta)),
$$

and the projected volume form transforms as

$$
d q \wedge d p=d \zeta \wedge d \eta
$$

This means that expectations of a function $f(q, p)$ can be evaluated (locally) as

$$
\mathbf{E}\{f\}=\int_{\mathcal{D}} f(q(\zeta), p(\zeta, \eta)) e^{-\beta \hat{H}(\zeta, \eta)} d \zeta \wedge d \eta,
$$

where the integration is understood as an integral over nonoverlapping local coordinate charts. Hence we consider the projected distribution

$$
\rho(\zeta, \eta)=Z^{-1} \exp (-\beta \hat{H}(\zeta, \eta)), \quad Z=\int_{\mathcal{D}} \exp (-\beta \hat{H}(\zeta, \eta)) d \zeta \wedge d \eta .
$$

For future reference we note from (35) and (32) that $\eta$ is mean-zero distributed in $\rho$, i.e., $\left\langle\eta_{i}\right\rangle=0$.

The generalization of the Langevin dynamics (15)-(16) to the constrained system (8)-(10) has been treated in [21]. Considering the phase space measure $\mu_{\mathcal{T} \mathcal{M}}$ of $\mathcal{T} \mathcal{M}$. The system which admits this measure as an invariant equilibrium measure is the following Langevin process with holomonic constraints:

$$
\begin{aligned}
d q & =M^{-1} p d t \\
d p & =-\nabla V(q) d t-\nabla g(q)^{T} \lambda d t-\gamma(q) M^{-1} p d t+\sigma(q) d W \\
0 & =g(q)
\end{aligned}
$$

where $W(t)$ is n-dimensional Wiener process, and $\gamma(q), \sigma(q)$ are $n \times n$ real matrices. The standard fluctuation-dissipation identity

$$
\sigma(q) \sigma(q)^{T}=\frac{2}{\beta} \gamma(q)
$$

has to be imposed such that the canonical distribution on the tangent bundle $\mathcal{T} \mathcal{M}$ with the phase space measure $\mu_{\mathcal{T} \mathcal{M}}$ is invariant under the dynamics $(36)-(38)$.

In local coordinates, the Langevin dynamics (36)-(38) takes the following form:

$$
\begin{aligned}
& d \zeta=\nabla_{\eta} \hat{H} d t, \\
& d \eta=-\nabla_{\zeta} \hat{H} d t-\Gamma(\zeta) \nabla_{\eta} \hat{H} d t+\Sigma(\zeta) d W,
\end{aligned}
$$


where $\Sigma(\zeta)=\nabla \phi^{T} \sigma(\phi(\zeta))$ and the standard fluctuation-dissipation identity

$$
\Sigma(\zeta) \Sigma(\zeta)^{T}=\frac{2}{\beta} \Gamma(\zeta)
$$

is satisfied in order for the projected distribution (35) to be invariant under the dynamics of (40)-(41).

The Nosé-Hoover-Langevin dynamics extended with holonomic constraint read:

$$
\begin{aligned}
d q & =M^{-1} p d t, \\
d p & =-\nabla V(q) d t-\nabla g(q)^{T} \lambda d t+\xi p d t, \\
d \xi & =h(p) d t-\gamma \xi d t+\sigma d w, \\
0 & =g(q),
\end{aligned}
$$

where $\xi$ is an auxiliary thermostat variable, $w(t)$ is scalar Wiener process, $\sigma \in \mathbf{R}$, $\gamma=\alpha \sigma^{2} / 2$ and the function $h(p): \mathbf{R}^{n} \rightarrow \mathbf{R}$ has to be determined.

To find the function $h(\zeta, \eta)$ we ask that the extended projected distribution

$$
\hat{\rho}(q, p, \xi)=\rho(\zeta, \eta) \rho_{\alpha}(\xi),
$$

where $\rho_{\alpha}$ is defined in (21), be invariant under the Fokker-Planck equation. The calculation is given in Appendix A. We find that

$$
h(p)=\frac{1}{\alpha}\left(n-m-\beta p^{T} M^{-1} p\right)
$$

in generalized coordinates $(q, p)$. Note the difference between the constants $n$ of $h(p)$ in NHL dynamics without constraint (17)-(19) and $n-m$ of $h(p)$ in the NHL dynamics with constraint (43)-(46). This form is also applicable to the original Hoover thermostat applied to constrained systems.

The Stochastic Velocity Rescaling thermostat method with holomonic constraints reads:

$$
\begin{aligned}
d q & =M^{-1} p d t, \\
d p & =-\nabla V(q) d t-\nabla g(q)^{T} \lambda d t-\Psi(K) p d t+\sqrt{2 k_{B} T \Phi(K)} p d W, \\
0 & =g(q),
\end{aligned}
$$

where $W(t)$ is a scalar Wiener processes, and $\Phi, \Psi$ are related by (see Appendix A):

$$
\Psi(K)=\left(2 K-(1+n-m) k_{B} T\right) \Phi(K)-2 k_{B} T K \frac{d \Phi}{d K} .
$$

Note the difference in the constants $1+n$ in the SVR dynamics without constraints (22)-(23) and $1+n-m$ for the dynamics with constraints (49)-(51). For the SVR dynamics with constraints the original proposal of Bussi et al. $[1,2]$ transforms to

$$
\Phi(K)=\frac{\gamma^{\prime \prime}}{2 K}, \quad \text { so that } \quad \Psi(K)=\left(1-\frac{n-m-1}{2 K} k_{B} T\right) \gamma^{\prime \prime} .
$$




\subsection{Numerical methods}

All of the methods mentioned above are easily implemented in the constrained setting using ideas of geometric integration (splitting methods). For a discussion of numerical methods for Langevin dynamics, see [27]. The numerical implementations are discussed in Appendix B.

\section{Relative efficiencies of NHL and Langevin with constraints}

Our approach to investigating the relative efficiencies of the different schemes is to determine the Maclaurin expansion of the velocity autocorrelation function (VAF), comparing the asymptotic convergence of this expansion in the limit of small correlation time $\tau$. All methods are expected to recover the correct (de-)correlation in the limit $\tau \rightarrow \infty$. The intermediate time $0 \ll \tau \ll \infty$ is also interesting, but does not easily yield to analysis. However it is hard to imagine that a method can be accurate for intermediate correlation times if it is inaccurate in the limit $\tau \rightarrow 0$ studied here.

The analysis closely follows that of [19] and the results are analogous to the unconstrained case. For that reason we consider here only the NHL and Langevin methods, and refer the reader to [19] for the SVR method.

Following Leimkuhler et al. [19],

$$
\begin{gathered}
F(t):=\frac{1}{F_{0}} \mathbf{E}_{e q}\left\{p(0)^{T} M^{-1} p(t)\right\}=\frac{1}{F_{0}} \mathbf{E}_{e q}^{t}\left\{p(0)^{T} M^{-1} p\right\}, \\
F_{0}:=\mathbf{E}_{e q}\left\{p(0)^{T} M^{-1} p(0)\right\},
\end{gathered}
$$

in the measure (14) for both constrained Langevin dynamics (36)-(38) and constrained NHL dynamics (43)-(46). These are compared with the expansion arising from the microcanonical dynamics (8)-(10). In [19] it was shown that in the limit $t \searrow 0$, Langevin dynamics decorrelates linearly in $t$ whereas NHL dynamics decorrelates as $t^{2}$, as does the microcanonical dynamics. That is, in the limit $t \searrow 0$, NHL dynamics approaches microcanonical dynamics asymptotically.

We expand the VAF in Maclaurin series,

$$
F(t)=1+\left.t \frac{d F(t)}{d t}\right|_{t=0+}+\left.\frac{t^{2}}{2} \frac{d^{2} F(t)}{d t^{2}}\right|_{t=0+}+O\left(t^{3}\right), \quad(t>0)
$$

and for comparison we compute the first and the second derivatives of $F$ for the Hamiltonian, Langevin and NHL dynamics.

\subsection{Hamiltonian dynamics}

We compute the first derivatives of the VAF for the constrained Hamiltonian dynamics (8)-(10) (without a thermostat). Multiplying equation (9) by $M^{-1} p(0)$ and taking the expectation with respect to the equilibrium measure (14) we obtain

$$
\begin{aligned}
\frac{d F_{\text {Ham }}(t)}{d t} & =\frac{1}{F_{0}} \mathbf{E}_{e q}^{t}\left\{p(0)^{T} M^{-1} \frac{d p}{d t}\right\} \\
& =-\frac{1}{F_{0}} \mathbf{E}_{e q}^{t}\left\{p(0)^{T} M^{-1} \nabla V(q)\right\}-\frac{1}{F_{0}} \mathbf{E}_{e q}^{t}\left\{p(0)^{T} M^{-1} \nabla g(q)^{T} \lambda\right\} \quad(t>0) .
\end{aligned}
$$


Using the canonical transformation (30)-(31) the first expectation value is

$$
\begin{gathered}
\mathbf{E}_{e q}^{t}\left\{p(0)^{T} M^{-1} \nabla V(q)\right\}= \\
\mathbf{E}_{e q}^{t}\left\{\eta(0)^{T}\left(\nabla \phi(0)^{T} \nabla \phi(0)\right)^{-T} \nabla \phi(0)^{T} M^{-1} \nabla \phi^{T} \nabla V(\phi(\zeta))\right\}=0,
\end{gathered}
$$

since $\eta$ in each component is mean-zero distributed in (35). Taking the limit $t \searrow 0$, the second expectation value is equal to zero since $M^{-1} p(0)$ belongs to the tangent space $T_{q(0)} \mathcal{M}$. Hence we obtain

$$
\left.\frac{d F_{\text {Ham }}(t)}{d t}\right|_{t=0+}=0
$$

We compute the second derivative of the VAF. Differentiating equation (9) with respect to $t$, multiplying by $M^{-1} p(0)$ and taking the expectation value with respect to the equilibrium measure (14) we obtain

$$
\begin{aligned}
\frac{d^{2} F_{H a m}(t)}{d t^{2}} & =\frac{1}{F_{0}} \mathbf{E}_{e q}^{t}\left\{p(0)^{T} M^{-1} \frac{d^{2} p}{d t^{2}}\right\}=-\frac{1}{F_{0}} \mathbf{E}_{e q}^{t}\left\{p(0)^{T} M^{-1} \operatorname{Hess}_{q}(\tilde{H}) \frac{d q}{d t}\right\} \\
& =-\frac{1}{F_{0}} \mathbf{E}_{e q}^{t}\left\{p(0)^{T} M^{-1} \operatorname{Hess}_{q}(\tilde{H}) \nabla_{p} \tilde{H}\right\} \quad(t>0),
\end{aligned}
$$

where $\operatorname{Hess}_{q}(\tilde{H})$ stands for Hessian matrix of the constrained Hamiltonian function (11) with respect to $q$. In the limit $t \searrow 0$, the term in braces is positive definite, so

$$
\left.\frac{d^{2} F_{\text {Ham }}(t)}{d t^{2}}\right|_{t=0+} \neq 0
$$

(in fact this term is strictly negative). Hence the Maclaurin expansion of VAF of the Hamiltonian dynamics is

$$
F_{\text {Ham }}(t)=1+\left.\frac{t^{2}}{2} \frac{d^{2} F_{\text {Ham }}(t)}{d t^{2}}\right|_{t=0+}+O\left(t^{3}\right) \quad(t>0) .
$$

\subsection{Langevin dynamics}

We compute the first derivative of the VAF for the Langevin dynamics. Multiplying equation (37) by $M^{-1} p(0)$, taking the expectation with respect to the equilibrium measure (14) we obtain, since $p(0)$ and $d W$ are statistically independent if $t>0$,

$$
\begin{aligned}
\frac{d F_{L D}(t)}{d t} & =\frac{1}{F_{0}} \mathbf{E}_{e q}^{t}\left\{p(0)^{T} M^{-1} \frac{d p}{d t}\right\} \\
& =\frac{d F_{\text {Ham }}(t)}{d t}-\frac{1}{F_{0}} \mathbf{E}_{e q}^{t}\left\{\frac{\beta}{2} p(0)^{T} M^{-1} \sigma(q) \sigma(q)^{T} M^{-1} p\right\} \quad(t>0) .
\end{aligned}
$$

Taking the limit $t \searrow 0$, we obtain

$$
\left.\frac{d F_{L D}(t)}{d t}\right|_{t=0+}=-\frac{1}{F_{0}} \mathbf{E}_{e q}\left\{\frac{\beta}{2} p(0)^{T} M^{-1} \sigma(q(0)) \sigma(q(0))^{T} M^{-1} p(0)\right\}=-\hat{\gamma} \neq 0
$$


and the Maclaurin expansion of the VAF for Langevin dynamics is

$$
F_{L D}(t)=1-\hat{\gamma} t+O\left(t^{2}\right) \quad(t>0)
$$

with explicit dependence on the parameter $\hat{\gamma}$. Since Hamiltonian dynamics is the same as Langevin dynamics with $\sigma(q)=0$, which in turn implies $\hat{\gamma}=0$, the error in $F(t)$ due to the use of Langevin dynamics rather than Hamiltonian dynamics is

$$
\Delta_{L D} F(t):=F_{L D}(t)-F_{\text {Ham }}(t)=-\hat{\gamma} t+O\left(t^{2}\right) \quad(t>0) .
$$

Thus for small $t$ the magnitude of the error is $\hat{\gamma} t$.

\subsection{The NHL dynamics}

The NHL thermostat depends on the variable $\xi$. The following computations are done with respect to the extended equilibrium hybrid (Gibbs) distribution (14). We begin by computing the first derivative of the VAF for the NHL dynamics. Multiplying the equation (37) by $M^{-1} p(0)$, taking the equilibrium expectation and dividing by $d t$ we obtain

$$
\begin{aligned}
\frac{d F_{N H L}(t)}{d t} & =\frac{1}{F_{0}} \mathbf{E}_{e q}^{t}\left\{p(0)^{T} M^{-1} \frac{d p}{d t}\right\} \\
& =\frac{d F_{\text {Ham }}(t)}{d t}+\frac{1}{F_{0}} \mathbf{E}_{e q}^{t}\left\{\xi p(0)^{T} M^{-1} p\right\} \quad(t>0) .
\end{aligned}
$$

Taking the limit $t \searrow 0$, we find that

$$
\left.\frac{d F_{N H L}(t)}{d t}\right|_{t=0+}=\frac{1}{F_{0}} \mathbf{E}_{e q}\left\{\xi(0) p(0)^{T} M^{-1} p(0)\right\}=0 .
$$

The result follows from the fact that $\xi$ is mean-zero normally distributed in the extended measure (47). Thus, the Maclaurin series of VAF for NHL dynamics begins with a quadratic term, for which we need the second derivative of $F(t)$ at $t=0$.

We define the function

$$
y:=-\nabla_{q} \tilde{H}+\xi p
$$

such that the equation (44) for $d p$ can be written $d p=y d t$. Differentiating $y$ by the Itō-Doeblin formula and using the equations (43)-(45) we obtain

$$
\begin{aligned}
d y= & -\operatorname{Hess}_{q}(\tilde{H}) d q+\xi d p+p d \xi \\
= & -\operatorname{Hess}_{q}(\tilde{H}) \nabla_{p} \tilde{H} d t+\xi\left(-\nabla_{q} \tilde{H}+\xi p\right) d t \\
& +p(h(p) d t-\gamma \xi d t+\sigma d w) \quad(t>0) .
\end{aligned}
$$

Hence

$$
\begin{aligned}
\frac{d^{2} F_{N H L}(t)}{d t^{2}} & =\frac{1}{F_{0}} \frac{d}{d t} \mathbf{E}_{e q}^{t}\left\{p(0)^{T} M^{-1} y\right\} \\
& =\frac{1}{F_{0}} \mathbf{E}_{e q}^{t}\left\{p(0)^{T} M^{-1} \frac{d y}{d t}\right\}, \quad(t>0) .
\end{aligned}
$$

Since $p(0)$ and $d w(t)$ are statistically independent if $t>0$,

$$
\begin{aligned}
\frac{d^{2} F_{N H L}(t)}{d t^{2}}= & \frac{d^{2} F_{H a m}(t)}{d t^{2}}-\frac{1}{F_{0}} \mathbf{E}_{e q}^{t}\left\{\xi p(0)^{T} M^{-1}\left(\nabla_{q} \tilde{H}+\gamma p\right)\right\} \\
& +\frac{1}{F_{0}} \mathbf{E}_{e q}^{t}\left\{\xi^{2} p(0)^{T} M^{-1} p\right\}+\frac{1}{F_{0}} \mathbf{E}_{e q}^{t}\left\{p(0)^{T} M^{-1} p h(p)\right\} \quad(t>0) .
\end{aligned}
$$




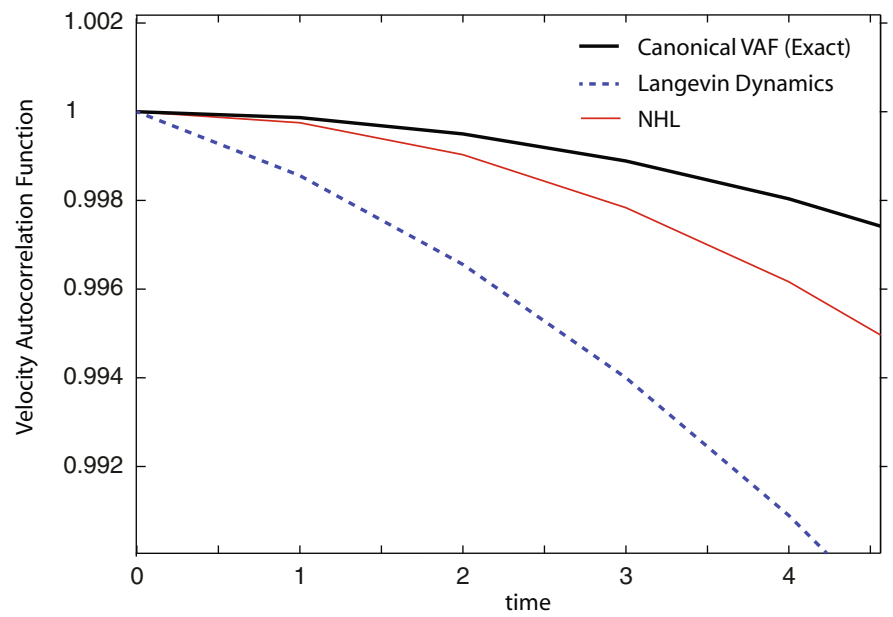

Fig. 1. Convergence of velocity auto-correlation functions for: microcanonical simulation, NHL method, and Langevin method, in the limit of small t.

Taking the limit $t \searrow 0$ and omitting the terms which turn out to be zero we find that

$$
\begin{aligned}
& \left.\frac{d^{2} F_{N H L}(t)}{d t^{2}}\right|_{t=0+}=\left.\frac{d^{2} F_{\text {Ham }}(t)}{d t^{2}}\right|_{t=0+}+\frac{1}{F_{0}} \mathbf{E}_{e q}^{t}\left\{\xi(0)^{2} p(0)^{T} M^{-1} p(0)\right\} \\
& +\frac{1}{F_{0}} \mathbf{E}_{e q}^{t}\left\{p(0)^{T} M^{-1} p(0) h(p(0))\right\} \\
& =\left.\frac{d^{2} F_{\text {Ham }}(t)}{d t^{2}}\right|_{t=0+}+C(\alpha),
\end{aligned}
$$

where $C(\alpha) \rightarrow 0$ when $\alpha \rightarrow \infty$. This thermostat reduces to the Hamiltonian dynamics in the limit $\alpha \rightarrow \infty$, since in this limit $h(p) \rightarrow 0$ and the Gaussian distribution for $\xi$ converges to the delta function $\delta(\xi)$, such that the expectation value of $\xi(0)^{2}$ is equal to zero.

Thus

$$
\Delta_{N H L} F(t):=F_{N H L}(t)-F_{H a m}(t)=\frac{1}{2} C(\alpha) t^{2}+O\left(t^{3}\right) \quad(t>0),
$$

i.e., in the limit $t \searrow 0$, NHL dynamics approaches microcanonical dynamics asymptotically. This asymptotic behaviour at small $t$ is illustrated in Fig. 1 for a double pendulum.

\section{Treatment of a flexible constraint}

Let us briefly recount the observations of $[9,10,12,13,15,16,25]$ regarding the statistical mechanics of systems in which a stiff restraining term is replaced by a holonomic constraint. To illustrate the discussion, consider, as in [25], a Hamiltonian

$$
H=H_{0}(q, p)+U_{\varepsilon}(q), \quad H_{0}(q, p)=\frac{p^{T} M^{-1} p}{2}+U(q), \quad U_{\varepsilon}(q)=\frac{1}{2 \varepsilon} g^{2}(q)
$$


where $g: \mathbf{R}^{n} \rightarrow \mathbf{R}$ is a function of the position variables and $\varepsilon$ is a (small) parameter. The equations of motion are

$$
\begin{aligned}
& \frac{d q}{d t}=M^{-1} p, \\
& \frac{d p}{d t}=-\nabla U(q)-\varepsilon^{-1} \nabla g(q)^{T} g(q) .
\end{aligned}
$$

In the limit $\varepsilon \rightarrow 0$, the equations reduce to constrained Euler-Lagrange equations

$$
\begin{aligned}
\frac{d q}{d t} & =M^{-1} p, \\
\frac{d p}{d t} & =-\nabla U(q)-\nabla g(q)^{T} \lambda, \\
0 & =g(q) .
\end{aligned}
$$

If we denote the solution of the flexible system by $\left(q_{\varepsilon}, p_{\varepsilon}\right)$ the suggestion is that

$$
\varepsilon^{-1} g\left(q_{\varepsilon}(t)\right) \sim \lambda .
$$

This simple analysis appears to justify replacing the stiff restraint by the constrained alternative, but the situation is a little more complicated. Let us assume that our original restrained system is modelled at a prescribed temperature $T$. We expect, assuming ergodicity, that some energy is present in the degree of freedom corresponding to the transverse (vibrational) motion. In the linearly restrained case, i.e. if $g(q)=\gamma \cdot q-\delta$, for some vector $\gamma$ and scalar $\delta$, the energy of restraint is quadratic and we easily justify

$$
\varepsilon^{-1}\left\langle g^{2}\left(q_{\varepsilon}\right)\right\rangle \sim k_{B} T .
$$

(It might be assumed that a similar relation holds for more general systems as long as the constraints are sufficiently smooth.) Thus some energy is present in the restraint, of fixed amount and independent of $\varepsilon$. In the constrained case there is no transverse energy at all. Thus there is a gap between the two models, and this will lead to incorrect calculation of statistical quantities when the constrained model is substituted for the unconstrained one. In essence, this means that the stiffer the restraint, the faster the restraint oscillates.

The idea of Van Kampen [15] and Fixman [10] was to "average out" over the fast vibrational motion, computing the free energy of the remaining degrees of freedom in the presence of this rapidly fluctuating auxiliary variable. Then it turns out that the modification of configurational statistics needed in order to compensate for the vibrational degrees of freedom can be modelled by the incorporation of the simple potential energy correction term, often termed the Fixman potential. The modified constrained system is then simply

$$
\begin{aligned}
\frac{d q}{d t} & =M^{-1} p, \\
\frac{d p}{d t} & =-\nabla U(q)-\nabla U_{\mathrm{Fix}}(q)-\nabla g(q)^{T} \lambda, \\
0 & =g(q),
\end{aligned}
$$

where

$$
U_{\text {Fix }}(q)=k_{B} T \ln \|\nabla g\| .
$$


The observation is that the canonical statistical mechanics of this system will provide configurational averages which are corrected for the constraining approximation. That is, canonical averages of functions of the positions of the Fixman- adjusted constrained system will correspond, in the limit $\varepsilon \rightarrow 0$, to the corresponding averages taken in the unconstrained system. (This relationship has recently been explored in detail by C. Hartmann [26].) Hence the corrected model can be used as a foundation for configurational sampling. It is important to note, however, that even the stationary averages of functions of momenta-let alone autocorrelation functions or diffusion constants - will be incorrect with or without the Fixman term.

This raises an interesting question. If our goal is to compute some dynamical quantities, how can we achieve this in the setting of constrained dynamics? Clearly we have no hope of calculating accurate dynamics that heavily depends on the vibrational (transverse to the constraint manifold) degrees of freedom, unless we are prepared to properly model this. But what if the function of interest is, for example, a long term rearrangement involving some coarsened degrees of freedom such as backbone dihedral angles or tertiary structural characteristics in a biomolecule, or order parameters or end-to-end stretch in a polymer? Then one may still hope that the dynamics of the Fixman system will reflect some of the dynamical properties of interest. However, there is an additional complication: the thermostat! The Fixman system itself only makes sense if it is implemented within a framework of canonical molecular dynamics, implying the use of a thermostat. The thermostat will itself complicate the picture in general, and distort the dynamics of the model. Thus we see an added motivation for a gentle thermostat in the setting of soft constraints.

\section{Numerical experiment}

In this section, we compare a number of the mentioned methods for the problems of calculating equilibrium distributions and dynamics of a small planar constraint chain. We begin with the model of an $N$-particle chain defined by the Hamiltonian

$$
H=\frac{1}{2} \sum_{i=1}^{N}\left\|p_{i}\right\|^{2}+\frac{1}{2 \varepsilon} \sum_{i=1}^{N}\left(\left\|q_{i}-q_{i-1}\right\|-1\right)^{2}+\sum_{i=0}^{N} \sum_{j=i+2}^{N} \phi_{L J}\left(\left\|q_{i}-q_{j}\right\|\right)
$$

where $q_{i} \in \mathbf{R}^{2},\|\cdot\|$ represents Euclidean 2-norm and $\phi_{L J}$ is the Lennard-Jones potential, acting between all atom pairs except those sharing a bond. We define $q_{0} \equiv 0$ in all summations. For appropriately scaled initial conditions, when $\varepsilon$ is driven to zero the system assumes the constrained form with $N-1$ constraints of the form $g_{i}(q)=\left\|q_{i+1}-q_{i}\right\|^{2}-1=0[16]$. Fixman forces were calculated for this model and are given (for pedagogical purposes) in Appendix C. We were interested in the comparison of both sampling and dynamics of the different constrained methods with those of the unconstrained model. We used a small value $\varepsilon=10^{-4}$ for the restraint, making a stiff spring which introduced an additional numerical challenge due to stability of the numerical method. For simplicity we worked with a chain of length 4 which gave sufficiently interesting behaviour.

We first compare the equilibrium distributions obtained by the various methods. We chose to compute the end-to-end distance (chain extension) $R=\left\|q_{N}-q_{0}\right\|$ as observable. The distributions (at $k_{B} T=2$ ) for $R$ were computed with each method. These are shown in Fig. 2. For the calculation in the unconstrained case we used small stepsizes $\Delta t=10^{-4}$ and in the constrained case $\Delta t=10^{-2}$. All long time simulations were run on the interval $t \in\left[0,10^{6}\right]$. 

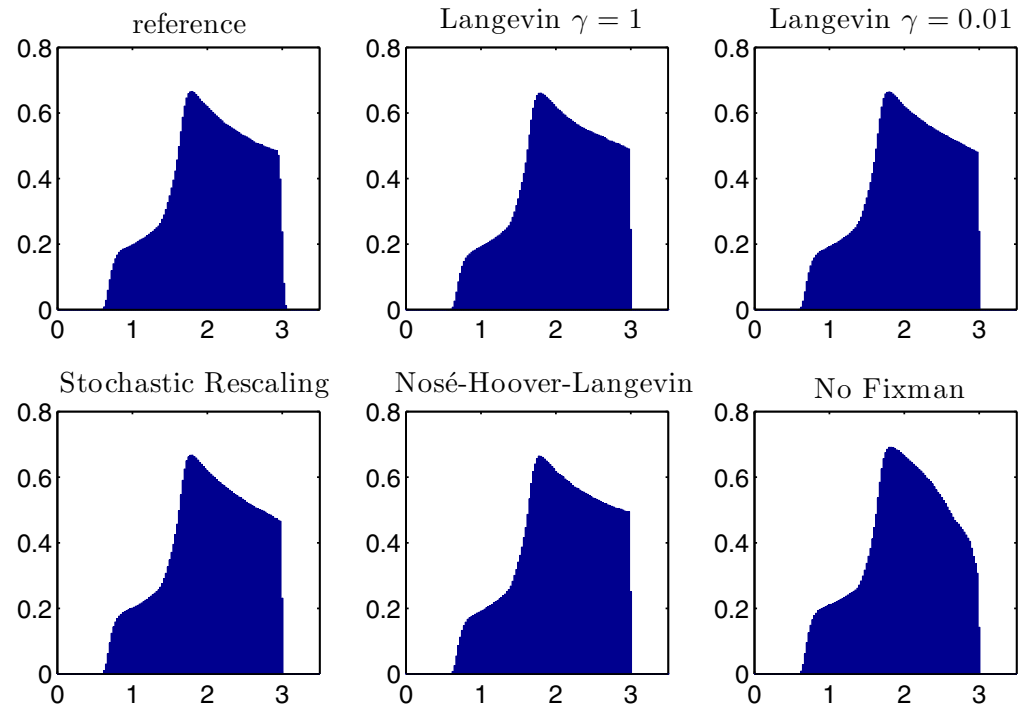

Fig. 2. PDFs of the end-to-end distance $R=\left\|q_{N}-q_{0}\right\|$ for the chain model (85), using stiff restraints (top left) and constraints: Langevin with strong $\gamma=1$ (top center) and weak $\gamma=0.01$ (top right) thermostating, SVR (bottom left) and NHL (bottom center) methods. The bottom right PDF illustrates the necessity of the Fixman correction.

As shown in Fig. 2 all thermostat methods (Langevin, SVR, and NHL) produced PDFs that were essentially identical to the reference distribution, over a wide range of parameter values $\gamma$. This indicates that the methods are ergodic in the desired measure, and that the Fixman force is effective in correcting the distribution to that of the stiff restrained case. On the contrary, the lower right subplot in Fig. 2 includes a distribution computed without Fixman correction (using NHL). The distribution is altered, especially for large extension lengths $R$, illustrating the necessity of the correction term.

Next, we considered the approximation of autocorrelation functions using the constrained thermostat methods. The analysis of Sect. 4 showed that the NHL method reproduces the velocity auto-correlation function to second order in $\tau$ as $\tau \rightarrow 0$. It is clear from the derivation that this analysis is specific to velocity auto-correlations. In this section we instead consider a different auto-correlation function, i.e. the relaxation of the difference of the end-to-end distance from its mean value (calculated by averaging over a trajectory),

$$
\varphi(\tau)=E_{\mathrm{eq}}(R(\tau)-\bar{R})(R(0)-\bar{R}) .
$$

We will investigate numerically the accuracy of the NHL, SVR and Langevin dynamics for this function, following the common practice of evaluating the expectations through long time averaging, relying on the assumption of ergodicity. Leimkuhler et al. [19] derive a first order analysis of the rate of convergence to the canonical measure for NHL, SVR and Langevin dynamics. The analysis in [19] indicates a rate of convergence for all methods proportional to the dissipation parameter $\gamma$ and to the number of degrees of freedom. In our simulations we choose, for the Langevin and SVR thermostats, $\gamma=0.25$; and for the NHL thermostat, $\gamma=1, \alpha=12$. For these values, all methods approach the desired temperature at approximately the same rate (slope $-1 / 2$ ), as shown in Fig. 3 . We then investigate the degree to which the associated auto-correlation function for $R$ approximates that of the reference curve. 


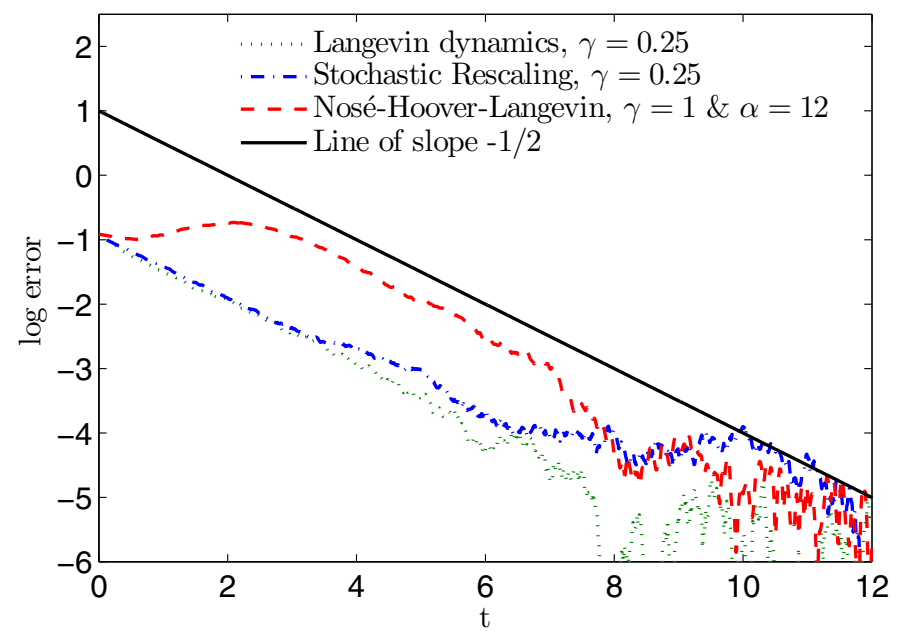

Fig. 3. Convergence to temperature $k_{B} T=2$ for the Langevin, SVR, and NHL methods, averaged over a $10^{4}$-member ensemble with initial temperature $k_{B} T=2.4$.

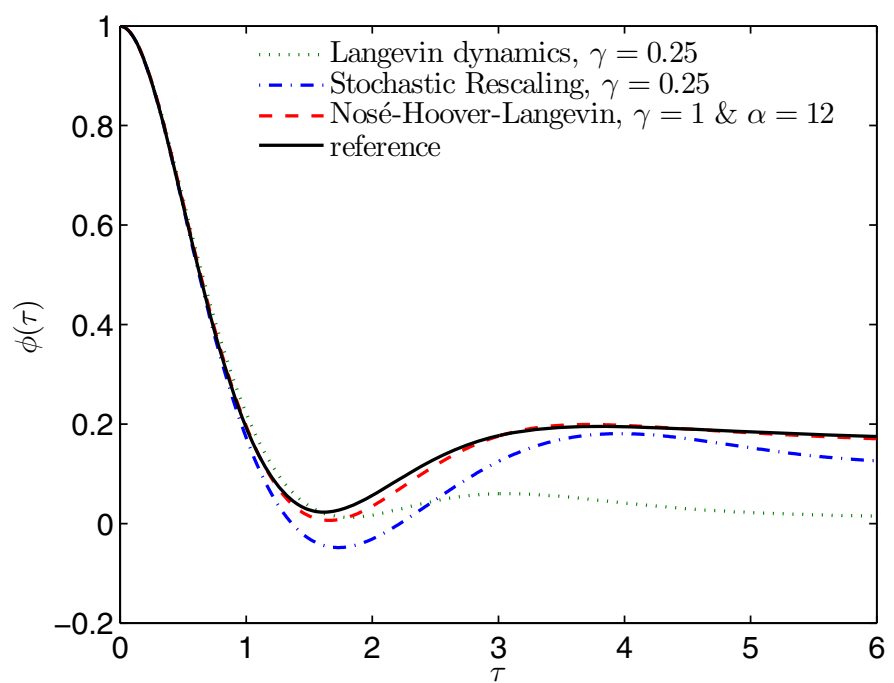

Fig. 4. Autocorrelation of the end-to-end distance as a function of time using Langevin, SVR, and NHL thermostats. The reference curve was computing using constant energy simulations from a canonically distributed ensemble.

The reference solution was computed using a $10^{6}$-member ensemble, canonically distributed initial conditions, and Hamiltonian (constant energy) dynamics with a stiff restraint $\left(\varepsilon=10^{-4}\right)$.

In Fig. 4 we see that Langevin dynamics with $\gamma=0.25$, although giving a good sampling of the equilibrium state, completely misses the dynamics of the system beyond the first trough. For smaller values of $\gamma$ the results can be improved somewhat, but in no case was the autocorrelation function well approximated on the given interval. Both the SVR and NHL methods capture the qualitative shape of the autocorrelation function, with NHL approximating the reference solution very closely over the whole interval. 


\section{Conclusion}

In this article, we have presented an overview of stochastic-dynamical thermostatting methods for constrained molecular modelling. We have shown that these methods have properties analogous to those of the unconstrained case. The Nosé-Hoover-Langevin method and the Stochastic Velocity Rescaling method were shown to weakly perturb the dynamics of the system. An application where thermostats are probably essential is in the evolution of constrained systems in the presence of a thermodynamic correction, and for these problems we have shown that the NHL and SVR thermostats with Fixman correction can provide improved accuracy in the autocorrelation function compared to a stiffly restrained model.

\section{Appendix A: Constrained stochastic thermostats}

In this appendix, we provide the derivations of the constrained forms of thermostat dynamics for the NHL and SVR methods, by introducing local coordinates on the constraint manifold.

We write down the equations (43)-(44) in local chart coordinates by differentiating the relations $(30)-(31)$.

$$
\begin{aligned}
d q & =\nabla \phi d \zeta, \\
\nabla \phi d \zeta & =\left(M^{-1} \nabla \phi\right)\left(\nabla \phi^{T} \nabla \phi\right)^{-1} \eta d t .
\end{aligned}
$$

We multiply both sides by $\nabla \phi^{T}$ and invert matrix $\nabla \phi^{T} \nabla \phi$ :

$$
\begin{aligned}
\left(\nabla \phi^{T} \nabla \phi\right) d \zeta & =\left(\nabla \phi^{T} M^{-1} \nabla \phi\right)\left(\nabla \phi^{T} \nabla \phi\right)^{-1} \eta d t \\
d \zeta & =\left(\nabla \phi^{T} \nabla \phi\right)^{-1}\left(\nabla \phi^{T} M^{-1} \nabla \phi\right)\left(\nabla \phi^{T} \nabla \phi\right)^{-1} \eta d t \\
d \zeta & =\nabla_{\eta} \hat{H} d t .
\end{aligned}
$$

Similarly,

$$
d p=\nabla \phi\left(\nabla \phi^{T} \nabla \phi\right)^{-1} d \eta+d\left(\nabla \phi\left(\nabla \phi^{T} \nabla \phi\right)^{-1}\right) \eta .
$$

We multiply both sides by $\nabla \phi^{T}$ and use property $\nabla \phi^{T} p=\eta$ to find $d \eta$ :

$$
\begin{aligned}
\nabla \phi^{T} d p & =d \eta+\nabla \phi^{T} d\left(\nabla \phi\left(\nabla \phi^{T} \nabla \phi\right)^{-1}\right) \eta \\
d \eta & =-\nabla \phi^{T} d\left(\nabla \phi\left(\nabla \phi^{T} \nabla \phi\right)^{-1}\right) \eta+\nabla \phi^{T} d p \\
d \eta & =-\nabla \phi^{T} d\left(\nabla \phi\left(\nabla \phi^{T} \nabla \phi\right)^{-1}\right) \eta-\nabla \phi^{T} \nabla V(\phi(\zeta)) d t+\xi \eta d t \\
d \eta & =\left[-D(\zeta)\left(\eta, M^{-1} \nabla \phi\left(\nabla \phi^{T} \nabla \phi\right)^{-1} \eta\right)-\nabla \phi^{T} \nabla V(\phi(\zeta))+\xi \eta\right] d t \\
d \eta & =-\nabla_{\zeta} \hat{H} d t+\xi \eta d t
\end{aligned}
$$

where $D(\zeta)$ is the symmetric three-tensor (Hessian) of partial derivative of $\nabla \phi\left(\nabla \phi^{T} \nabla \phi\right)^{-1}$, whose contraction is denoted $D(\zeta)(\cdot, \cdot)$. Equation (45) simply takes the following form:

$$
d \xi=h(\zeta, \eta) d t-\gamma \xi d t+\sigma d w
$$

To find the function $h(\zeta, \eta)$ we ask that the extended projected distribution (47) be invariant under the Fokker-Planck equation. We find that

$$
h(\zeta, \eta)=\frac{1}{\alpha}\left(\nabla \cdot \eta-\beta \nabla_{\eta} \hat{H} \cdot \eta\right)=\frac{1}{\alpha}\left(n-m-\beta \nabla_{\eta} \hat{H} \cdot \eta\right) .
$$


The NHL method for the equations in the local chart coordinates (90), (96) and (97) is ergodic in the extended projected measure (47) whenever the Lie algebra generated by $\eta$ and $\nabla_{\zeta} \hat{H}$ spans $\mathbf{R}^{n-m}$.

Since

$$
\begin{aligned}
\nabla_{\eta} \hat{H} \cdot \eta & =\left(\left(\nabla \phi^{T} \nabla \phi\right)^{-1}\left(\nabla \phi^{T} M^{-1} \nabla \phi\right)\left(\nabla \phi^{T} \nabla \phi\right)^{-1} \eta\right) \cdot \eta \\
& =\left(M^{-1} \nabla \phi\left(\nabla \phi^{T} \nabla \phi\right)^{-1} \eta\right) \cdot\left(\nabla \phi\left(\nabla \phi^{T} \nabla \phi\right)^{-1} \eta\right) \\
& =p^{T} M^{-1} p
\end{aligned}
$$

we find that

$$
h(p)=\frac{1}{\alpha}\left(n-m-\beta p^{T} M^{-1} p\right)
$$

in generalized coordinates $(q, p)$.

For the SVR thermostat (49)-(51), to find the relation between $\Phi$ and $\Psi$, we re-write the equations in local chart coordinates:

$$
\begin{aligned}
& d \zeta=\nabla_{\eta} \hat{H} d t \\
& d \eta=-\nabla_{\zeta} \hat{H} d t-\Psi(\hat{K}) \eta d t+\sqrt{2 k_{B} T \Phi(\hat{K})} \eta d W,
\end{aligned}
$$

where $\hat{K}$ is the kinetic energy in local coordinates. We ask that the projected distribution (35) be invariant under the Fokker-Planck equation. We find that functions $\Psi(\hat{K})$ and $\Phi(\hat{K})$ are related by

$$
\Psi(\hat{K})=\left(2 \hat{K}-(1+n-m) k_{B} T\right) \Phi(\hat{K})-2 k_{B} T \hat{K} \frac{d \Phi}{d \hat{K}} .
$$

In coordinates on $\mathbf{R}^{2 n}$ this relation reads:

$$
\Psi(K)=\left(2 K-(1+n-m) k_{B} T\right) \Phi(K)-2 k_{B} T K \frac{d \Phi}{d K} .
$$

The original proposal of Bussi et al. [1,2] for the SVR dynamics without constraints corresponds to the choice

$$
\Phi(K)=\frac{\gamma^{\prime \prime}}{2 K}, \quad \text { so that } \quad \Psi(K)=\left(1-\frac{n-1}{2 K} k_{B} T\right) \gamma^{\prime \prime},
$$

where $\gamma^{\prime \prime}$ is a positive constant (the constant $1 / 2 \gamma^{\prime \prime}$ is termed the 'relaxation time'). For the SVR dynamics with constraints the original proposal of Bussi et al. transforms to

$$
\Phi(K)=\frac{\gamma^{\prime \prime}}{2 K}, \quad \text { so that } \quad \Psi(K)=\left(1-\frac{n-m-1}{2 K} k_{B} T\right) \gamma^{\prime \prime} .
$$

\section{Appendix B: Aspects of time integration}

In this Appendix, we describe the numerical implementations of the Langevin, NHL and SVR thermostats with holonomic constraints. For all thermostat methods we adapt the RATTLE algorithm by splitting the system into deterministic and stochastic parts. RATTLE is a symmetric method and symplectic in the Hamiltonian limit. The stochastic part can be then solved, depending on the equations, analytically or numerically. 
We denote the time index with a superscript, and we consider a single time step, i.e. the map $\left(q^{0}, p^{0}\right) \mapsto\left(q^{1}, p^{1}\right)$.

We consider the Langevin thermostat, the Nosé-Hoover-Langevin thermostat and the Stochastic Velocity Rescaling thermostat equations with holonomic constraints (36)-(38), (43)-(46) and (49)-(51), respectively.

We split the right hand side vector field of (36)-(38) into a Hamiltonian part and a fluctuation-dissipation part acting only on the momentum. For simplicity, we restrict ourselves to constant, scalar $\sigma$. Hence the fluctuation-dissipation part reduces to the simple Ornstein-Uhlenbeck process, and since the mass matrix is typically diagonal the computation of the analytic solution of the Ornstein-Uhlenbeck process is cheap. Generalizations to constant and position dependent matrix $\sigma$ are straightforward by adapting the approach of [21].

The numerical method for the Langevin dynamics with holonomic constraints reads:

$$
\begin{aligned}
& \left\{\begin{array}{l}
\tilde{p}=\exp \left(-\gamma M^{-1} \frac{\tau}{2}\right) p^{0}+\sigma \sqrt{\frac{1-\exp \left(-\gamma M^{-1} \tau\right)}{2 \gamma}} M \Delta W^{0}-\frac{\tau}{2} \nabla g\left(q^{0}\right)^{T} \lambda_{0}, \\
0=\nabla g\left(q^{0}\right) M^{-1} \tilde{p},
\end{array}\right. \\
& \left\{\begin{array}{l}
q^{1}=q^{0}+\tau M^{-1} p^{1 / 2}, \\
p^{1 / 2}=\tilde{p}-\frac{\tau}{2} \nabla V\left(q^{0}\right)-\frac{\tau}{2} \nabla g\left(q^{0}\right)^{T} \lambda_{1}, \\
0=g\left(q^{1}\right), \\
\hat{p}=p^{1 / 2}-\frac{\tau}{2} \nabla V\left(q^{1}\right)-\frac{\tau}{2} \nabla g\left(q^{1}\right)^{T} \lambda_{2}, \\
0=\nabla g\left(q^{1}\right) M^{-1} \hat{p},
\end{array}\right. \\
& \left\{\begin{array}{l}
p^{1}=\exp \left(-\gamma M^{-1} \frac{\tau}{2}\right) \hat{p}+\sigma \sqrt{\frac{1-\exp \left(-\gamma M^{-1} \tau\right)}{2 \gamma} M \Delta W^{1}-\frac{\tau}{2} \nabla g\left(q^{1}\right)^{T} \lambda_{3},} \\
0=\nabla g\left(q^{1}\right) M^{-1} p^{1},
\end{array}\right.
\end{aligned}
$$

where $\tau$ is a time step and $\Delta W^{0}$ and $\Delta W^{1}$ are independently and identically distributed Gaussian random variables of mean 0 and covariance matrix $\operatorname{Id}_{n}$.

For the Nosé-Hoover-Langevin thermostat equations with holonomic constraints (43)-(46) we split the right hand side vector field in three parts, i.e., a Hamiltonian part, an external forcing and an Ornstein-Uhlenbeck process. Each resulting vector field is solved exactly, i.e. we solve

$$
\frac{d p}{d t}=\xi p
$$

for fixed value of $\xi$, and the scalar Ornstein-Uhlenbeck process

$$
d \xi=\gamma(\mu-\xi) d t+\sigma d w
$$

is also solved exactly for given Wiener increments. The numerical method for the Nosé-Hoover-Langevin thermostat equations with holonomic constraints reads:

$$
\left\{\tilde{p}=\exp \left(\frac{\tau}{2} \xi^{0}\right) p^{0}\right.
$$




$$
\begin{gathered}
\left\{\begin{array}{l}
q^{1}=q^{0}+\tau M^{-1} p^{1 / 2}, \\
p^{1 / 2}=\tilde{p}-\frac{\tau}{2} \nabla V\left(q^{0}\right)-\frac{\tau}{2} \nabla g\left(q^{0}\right)^{T} \lambda_{1}, \\
0=g\left(q^{1}\right),
\end{array}\right. \\
\left\{\begin{array}{l}
\xi^{1}=\exp (-\gamma \tau) \xi^{0}+\frac{1}{\gamma} h\left(p^{1 / 2}\right)(1-\exp (-\gamma \tau))+\sigma \sqrt{\frac{1-\exp (-2 \gamma \tau)}{2 \gamma} \Delta w} \\
\left\{\begin{array}{l}
\hat{p}=p^{1 / 2}-\frac{\tau}{2} \nabla V\left(q^{1}\right)-\frac{\tau}{2} \nabla g\left(q^{1}\right)^{T} \lambda_{2}, \\
0=\nabla g\left(q^{1}\right) M^{-1} \hat{p},
\end{array}\right. \\
\left\{p^{1}=\exp \left(\frac{\tau}{2} \xi^{1}\right) \hat{p},\right.
\end{array}\right.
\end{gathered}
$$

where $\tau$ is a time step and $\Delta w \sim \mathcal{N}(0,1)$. Since the velocities $p^{0}$ and $\hat{p}$ belong to the tangent spaces $T_{q^{0}} \mathcal{M}$ and $T_{q^{1}} \mathcal{M}$, respectively, it follows that the velocities $\tilde{p}$ and $\hat{p}$ belong to the tangent spaces $T_{q^{0}} \mathcal{M}$ and $T_{q^{1}} \mathcal{M}$, respectively. Hence we do not need to perform additional projection of the velocities onto the tangent spaces.

The stochastic part of the Stochastic Velocity Rescaling thermostat equations (49)-(51) reads:

$$
d p=-\Psi(K) p d t+\sqrt{2 k_{B} T \Phi(K)} p d W .
$$

Shortly we will show that the solution of this differential equation only changes the scaling of $p$ and not its direction. Since the RATTLE step ensures $p \in T_{q} \mathcal{M}$, it is not necessary to introduce a Lagrange multiplier into (119).

We make the ansatz $p(t)=\alpha(t) p^{0}$, where $\alpha(t)$ is a scalar function. Substituting this solution into (119) gives the $\mathrm{SDE}$

$$
p^{0} d \alpha=-\Psi\left(\alpha^{2} K_{0}\right) \alpha p^{0} d t+\sqrt{2 k_{B} T \Phi\left(\alpha^{2} K_{0}\right)} \alpha p^{0} d W
$$

Since each term contains a factor $p^{0}$, we can omit it, leaving a scalar SDE for $\alpha$, and proving our assertion.

For simplicity, we consider the relation (53) between functions $\Psi(K)$ and $\Phi(K)$ of the original proposal of Bussi et al. for the systems with constraints such that SDE (120) takes the particular form

$$
d \alpha=-\left(\alpha-\frac{n-m-1}{\alpha K_{0}} k_{B} T\right) \gamma d t+\sqrt{\frac{2 k_{B} T \gamma}{K_{0}}} d W
$$

where $K_{0}=p_{0}^{T} M^{-1} p_{0}$. We note that this SDE has additive noise, making it more amenable to numerical integration than the equation for $K$ proposed in [1]. We solve it by splitting into a nonlinear term and an Ornstein-Uhlenbeck process, applied symmetrically about the RATTLE step. Alternatively one could use the exact solution given in [1], but for our experiments the splitting method with single Wiener process was found to be a cheap alternative. Furthermore, we observed no adverse effects 
from splitting errors. The numerical method reads:

$$
\begin{aligned}
& \left\{\begin{array}{l}
K_{0}=p^{0^{T}} M^{-1} p^{0}, \\
\tilde{\alpha}=\sqrt{\frac{n-m-1}{K_{0}} k_{B} T \gamma \tau+1} \\
\alpha=\exp \left(-\gamma \frac{\tau}{2}\right) \tilde{\alpha}+\sqrt{\frac{1-\exp (-\gamma \tau)}{K_{0}} k_{B} T \Delta W^{0}} \\
\tilde{p}=\alpha p^{0},
\end{array}\right. \\
& \begin{array}{c}
\left\{\begin{array}{l}
q^{1}=q^{0}+\tau M^{-1} p^{1 / 2}, \\
p^{1 / 2}=\tilde{p}-\frac{\tau}{2} \nabla V\left(q^{0}\right)-\frac{\tau}{2} \nabla g\left(q^{0}\right)^{T} \lambda_{1}, \\
0=g\left(q^{1}\right), \\
\hat{p}=p^{1 / 2}-\frac{\tau}{2} \nabla V\left(q^{1}\right)-\frac{\tau}{2} \nabla g\left(q^{1}\right)^{T} \lambda_{2}, \\
0=\nabla g\left(q^{1}\right) M^{-1} \hat{p},
\end{array}\right. \\
\left\{\begin{array}{l}
K_{0}=\hat{p}^{T} M^{-1} \hat{p}, \\
\tilde{\alpha}=\exp \left(-\gamma \frac{\tau}{2}\right)+\sqrt{\frac{1-\exp (-\gamma \tau)}{K_{0}}} k_{B} T \Delta W^{1} \\
\tilde{\alpha}=\sqrt{\frac{n-m-1}{K_{0}} k_{B} T \gamma \tau+\tilde{\alpha}^{2}}, \\
p^{1}=\alpha \hat{p},
\end{array}\right.
\end{array}
\end{aligned}
$$

where $\tau$ is a time step and $\Delta W^{0}, \Delta W^{1} \sim \mathcal{N}(0,1)$.

\section{Appendix C: Fixman forces for the chain model}

In this appendix, we give the detailed description of the Fixman potential (84) and force for the chain model considered in Sect. 6 .

The Fixman potential for the chain model is defined by

$$
U_{\mathrm{Fix}}(q)=\frac{k_{B} T}{2} \ln \operatorname{det}\left(\nabla g(q) \nabla g(q)^{T}\right),
$$

where $\nabla g(q)$ is the Jacobian matrix of constraint $g(q)$. The matrix product $A:=$ $\nabla g(q) \nabla g(q)^{T}$ is a tri-diagonal symmetric matrix. To find the Fixman force

$$
F_{\mathrm{Fix}}=-\nabla U_{\mathrm{Fix}}=-\frac{k_{B} T}{2 \operatorname{det}\left(\nabla g(q) \nabla g(q)^{T}\right)} \nabla \operatorname{det}\left(\nabla g(q) \nabla g(q)^{T}\right)
$$

we need to compute the gradient of the determinant [9]. Let $\operatorname{det} A$ be the $\operatorname{determinant}$ of the matrix $A$, then the determinant can be expressed as

$$
\operatorname{det} A=\sum_{i}^{N} a_{i, j} C_{i, j}
$$


for any $j=1, \ldots, N$, where $C_{i, j}=(-1)^{i+j} M_{i, j}$ is a so-called cofactor and $M_{i, j}$ is a minor. Note that for the symmetric matrices $C_{i, j}=C_{j, i}$.

The derivative of the determinant $\operatorname{det} A$ with respect to each component of position vector $q_{k}$ is

$$
\frac{\partial \operatorname{det} A}{\partial q_{k}}=\sum_{i, j} \frac{\partial \operatorname{det} A}{\partial a_{i, j}} \frac{\partial a_{i, j}}{\partial q_{k}}=\sum_{i, j} C_{i, j} \frac{\partial a_{i, j}}{\partial q_{k}} .
$$

For the chain model special care has to be taken when $k=1,2, N-2, N$. The Fixman forces for the chain model can be computed by the following formulas:

$$
\begin{aligned}
F_{\mathrm{Fix}}^{1}= & 2 C_{1,1} q_{1}+4 C_{2,2}\left(q_{1}-q_{2}\right)+2 C_{1,2}\left(2 q_{1}-q_{2}\right)+2 C_{2,3}\left(q_{3}-q_{2}\right) \\
F_{\mathrm{Fix}}^{2}= & 4 C_{2,2}\left(q_{2}-q_{1}\right)-2 C_{2,1} q_{1}+4 C_{3,3}\left(q_{2}-q_{3}\right) \\
& -2 C_{2,3}\left(q_{1}-2 q_{2}+q_{3}\right)+2 C_{3,4}\left(q_{4}-q_{3}\right) \\
F_{\mathrm{Fix}}^{k}= & 4 C_{k, k}\left(q_{k}-q_{k-1}\right)+2 C_{k, k-1}\left(q_{k-2}-q_{k-1}\right)+4 C_{k+1, k+1}\left(q_{k}-q_{k+1}\right) \\
& -2 C_{k, k+1}\left(q_{k-1}-2 q_{k}+q_{k+1}\right) \\
& +2 C_{k+1, k+2}\left(q_{k+2}-q_{k+1}\right), \quad k=2 \ldots N-2 \\
F_{\mathrm{Fix}}^{N-1}= & 4 C_{N-1, N-1}\left(q_{N-1}-q_{N-2}\right)+2 C_{N-1, N-2}\left(q_{N-3}-q_{N-2}\right) \\
& +4 C_{N, N}\left(q_{N-1}-q_{N}\right)-2 C_{N-1, N}\left(q_{N-2}-2 q_{N-1}+q_{N}\right) \\
F_{\mathrm{Fix}}^{N}= & 4 C_{N, N}\left(q_{N}-q_{N-1}\right)+2 C_{N, N-1}\left(q_{N-2}-q_{N-1}\right)
\end{aligned}
$$

where each vector $F_{\text {Fix }}^{k}$ must be multiplied by $-k_{B} T / 2 / \operatorname{det}\left(\nabla g(q) \nabla g(q)^{T}\right)$.

\section{References}

1. G. Bussi, D. Donadio, M. Parrinello, J. Chem. Phys. 126, 014101 (2007)

2. G. Bussi, M. Parrinello, Comp. Phys. Comm. 179, 26 (2008)

3. W.K. den Otter, W. Briels, J. Chem. Phys. 106, 13 (1997)

4. M. Cassandro, G. Ciccotti, V. Rosato, J.P. Ryckaert, Statistical mechanics of rigid systems: an atomic description, unpublished note

5. G. Ciccotti, J.P. Ryckaert, Comp. Phys. Reports 4, 346 (1986)

6. G. Ciccotti, R. Kapral, E. Vanden-Eijnden, Chem. Phys. Chem. 6, 1809 (2005)

7. E. Darve, Ph.D. thesis, Université Paris VI (1999)

8. W.E.W. Ren, E. Vanden-Eijnden, Phys. Rev. B 66, 052301 (2002)

9. M. Fixman, P. Nat. Acad. Sci. 71, 3050 (1974)

10. M. Fixman, J. Chem. Phys. 69, 1527 (1978)

11. J. Frank, G.A. Gottwald, J. Stat. Phys. 143, 715 (2011)

12. N. Go, H.A. Scheraga, J. Chem. Phys. 51, 4751 (1969)

13. N. Go, H.A. Scheraga, Macromolecules 9, 535 (1976)

14. A. Jones, B. Leimkuhler (2011) (submitted)

15. N.G. van Kampen, Appl. Sci. Res. 37, 67 (1981)

16. N.G. van Kampen, J.J. Lodder, Am. J. Phys. 52, 419 (1984)

17. B. Leimkuhler, S. Reich, Math. Comp. 63, 598 (1994)

18. B. Leimkuhler, Phys. Rev. E 81, 026703 (2010)

19. B. Leimkuhler, E. Noorizadeh, O. Penrose, J. Stat. Phys. 143, 921 (2011)

20. B. Leimkuhler, E. Noorizadeh, F. Theil, J. Stat. Phys. 135, 261 (2009)

21. T. Lelièvre, M. Rousset, G. Stoltz (preprint) (2010)

22. A. Samoletov, M.A.J. Chaplain, C.P. Dettmann, J. Stat. Phys. 128, 1321 (2007) 
23. J.A. Izaguirre, D.P. Catarello, J.M. Wozniak, R.D. Skeel, J. Chem. Phys. 114, 2090 (2001)

24. P. Minary, G.J. Martyna, M.E. Tuckerman, Phys. Rev. Lett. 93, 150201 (2004)

25. S. Reich, Physica D. 89, 28 (1995)

26. C. Hartmann, Constraints in Molecular Simulation 2010, Zaragosa, slides, http://neptuno.unizar.es/events/constraints2010/files/Carsten_Hartmann.pdf

27. S. Melchionna, J. Chem. Phys. 127, 044108 (2007) 